\title{
Plasma fatty acids and the risk of vascular disease and mortality outcomes in individuals with type 2 diabetes: results from the ADVANCE study
}

\author{
Katie Harris ${ }^{1} \cdot$ Megumi Oshima ${ }^{1,2} \cdot$ Naveed Sattar $^{3} \cdot$ Peter Würtz ${ }^{4} \cdot$ Min Jun ${ }^{1} \cdot$ Paul Welsh $^{3}$ • Pavel Hamet ${ }^{5}$. \\ Stephen Harrap ${ }^{6} \cdot$ Neil Poulter $^{7} \cdot$ John Chalmers $^{1} \cdot$ Mark Woodward $^{1,8,9}$
}

Received: 5 December 2019 / Accepted: 1 April 2020 / Published online: 8 May 2020

(C) The Author(s) 2020

\begin{abstract}
Aims/hypothesis This biomarker study aimed to quantify the association of essential and other plasma fatty acid biomarkers with macrovascular disease, microvascular disease and death in individuals with type 2 diabetes.

Methods A case-cohort study $(N=3576)$, including 654 macrovascular events, 341 microvascular events and 631 deaths during 5 years of (median) follow-up, was undertaken as a secondary analysis of the Action in Diabetes and Vascular Disease: Preterax and Diamicron Modified-Release Controlled Evaluation (ADVANCE) study (full details of the study design and primary endpoints of the ADVANCE trial and its case-cohort have been published previously). This current study considers new data: fatty acids measured from baseline plasma samples by proton NMR analysis. The fatty acids measured were $n-3$, docosahexaenoic acid (DHA), $n$ - 6 , linoleic acid, and polyunsaturated, monounsaturated and saturated fatty acids. HRs were modelled per SD higher (percentage) fatty acid. C statistics and continuous net reclassification improvement were used to test the added value of fatty acids compared with traditional cardiovascular risk factors.

Results After adjustment for traditional cardiovascular risk factors, an inverse association was observed for $n-3$ fatty acids and DHA with the risk of macrovascular events (HR [95\% CI]: 0.87 [0.80, 0.95] and 0.88 [0.81, 0.96], respectively, per 1 SD higher percentage), and for $n-3$ fatty acids with the risk of death (HR 0.91 [95\% CI 0.84, 0.99] per 1 SD higher percentage). Such associations were also evident when investigating absolute levels of fatty acids. There were no statistically significant associations between any fatty acids and microvascular disease after adjustment. However, there was limited improvement in the predictive ability of models when any fatty acid was added.
\end{abstract}

Katie Harris and Megumi Oshima contributed equally to this work.

Electronic supplementary material The online version of this article (https://doi.org/10.1007/s00125-020-05162-z) contains peer-reviewed but unedited supplementary material, which is available to authorized users.

\section{Katie Harris}

kharris@georgeinstitute.org.au

$\triangle$ Mark Woodward

mark.woodward@georgeinstitute.ox.ac.uk

1 The George Institute for Global Health, UNSW Sydney, Level 10, King George V Building, 83-117 Missenden Road, Camperdown, Sydney, NSW 2050, Australia

2 Department of Nephrology and Laboratory Medicine, Kanazawa University, Ishikawa, Japan

3 BHF Glasgow Cardiovascular Research Centre, Institute of Cardiovascular and Medical Sciences, University of Glasgow, Glasgow, UK
$4 \quad$ Nightingale Health Ltd, Helsinki, Finland

5 Centre de Recherche, Centre Hospitalier de l'Université de Montréal (CRCHUM), Montréal, QC, Canada

6 Department of Physiology, Royal Melbourne Hospital, University of Melbourne, Melbourne, VIC, Australia

7 Imperial Clinical Trials Unit, School of Public Health, Imperial College London, London, UK

8 The George Institute for Global Health, University of Oxford, 1st Floor, Hayes House, 75 George Street, Oxford OX1 2BQ, UK

9 Department of Epidemiology, Johns Hopkins University, Baltimore, MD, USA 


\section{Research in context}

\section{What is already known about this subject?}

- Circulating $n-3$ fatty acids and docosahexaenoic acid (DHA; a subtype of $n-3$ ), which are generally known as seafood-derived fatty acids, have been reported to be associated with a lower risk of cardiovascular disease, while there have been conflicting reports about the benefits of dietary intake of these fatty acids

- Limited studies have assessed the benefits of measuring circulating fatty acids in people with type 2 diabetes in ascertaining the risks of macro- and microvascular diseases

What is the key question?

- Are levels of plasma fatty acids associated with the risks of major clinical outcomes in people with type 2 diabetes?

What are the new findings?

- There were inverse associations of baseline plasma $n-3$ fatty acids and DHA with the risk of macrovascular events (HR [95\% Cl]: 0.87 [0.80, 0.95] and 0.88 [0.81, 0.96], respectively, per 1 SD higher percentage), and of $n-3$ fatty acids with the risk of death ( $\mathrm{HR} 0.91[95 \% \mathrm{Cl} 0.84,0.99]$ per $1 \mathrm{SD}$ higher percentage), even after adjustment for traditional cardiovascular risk factors

- No significant associations were observed between any fatty acids and the risk of microvascular events

How might this impact on clinical practice in the foreseeable future?

- These results support the cardioprotective effects of $n-3$ fatty acids and DHA and further merit testing the role of high-dose $n-3$ fatty acid supplementation in people with type 2 diabetes

Conclusions/interpretation Plasma $n-3$ fatty acids and DHA were found to be inversely associated with macrovascular disease, while $n-3$ fatty acids were also inversely associated with death. These results support the cardioprotective effects of $n-3$ fatty acids and DHA and further merit testing the role of high-dose supplementation with $n-3$ fatty acids in individuals with type 2 diabetes. Trial registration ClinicalTrials.gov NCT00145925.

Keywords Diabetes complications · Docosahexaenoic acid (DHA) $\cdot n$-3 fatty acids $\cdot$ Plasma Fatty acids $\cdot$ Type 2 diabetes

$\begin{array}{ll}\text { Abbreviations } & \\ \text { ADVANCE } & \begin{array}{l}\text { Action in Diabetes and Vascular Disease: } \\ \text { Preterax and Diamicron Modified-Release } \\ \text { Controlled Evaluation }\end{array} \\ \text { AHA } & \text { American Heart Association } \\ \text { CVD } & \text { Cardiovascular disease } \\ \text { DHA } & \text { Docosahexaenoic acid } \\ \text { EPA } & \text { Eicosapentaenoic acid } \\ { }^{1} \text { H-NMR } & \text { Proton NMR } \\ \text { LA } & \text { Linoleic acid } \\ \text { MUFA } & \text { Monounsaturated fatty acids } \\ \text { NRI } & \text { Net reclassification index } \\ \text { PUFA } & \text { Polyunsaturated fatty acids } \\ \text { REDUCE-IT } & \text { Reduction of Cardiovascular Events with } \\ & \text { Icosapent Ethyl-Intervention Trial } \\ \text { SFA } & \text { Saturated fatty acids }\end{array}$

\section{Introduction}

Type 2 diabetes is associated with a substantial risk of macrovascular disease, including coronary and cerebrovascular diseases; microvascular disease, including kidney disease and retinopathy; and premature death [1]. Early recognition of diabetes in its progression and initiation of an intervention are therefore needed for preventing such adverse long-term outcomes.

Fatty acids are vital nutrients which play regulatory roles in energy metabolism. The composition of fatty acids in blood can be affected by dietary intake [2] and has been reported to be involved in pathological mechanisms of various diseases such as insulin resistance, obesity, diabetes and atherosclerosis [3]. For several decades, clinical trials and population-based studies have attempted to determine the effects of dietary intake of fatty acids on vascular outcomes and mortality; however, the results remain 
controversial and inconsistent. Several meta-analyses of clinical trials have suggested that dietary intake of $n-3$ fatty acids such as docosahexaenoic acid (DHA) and eicosapentaenoic acid (EPA), which are generally known as seafood-derived fatty acids, had no, or at most a weak, protective effect on cardiovascular disease (CVD) and death in people with and without diabetes $[4,5]$. In contrast, a recent meta-analysis reported that $n-3$ supplementation was associated with a lower risk of CVD [6]. In particular, the recent Reduction of Cardiovascular Events with Icosapent Ethyl-Intervention Trial (REDUCE-IT) observed pronounced protective effects of high-dose ( $4 \mathrm{~g} /$ day) supplementation with $n-3$ fatty acids against cardiovascular outcomes among individuals with established CVD or with diabetes and other risk factors [7]. Based upon these results, the recent scientific statement from the American Heart Association (AHA) recommends the prescription of $n-3$ fatty acids, whether EPA+DHA or EPA only, at a dose of $4 \mathrm{~g} /$ day as an effective and safe treatment for reducing triacylglycerols among individuals with hypertriglyceridaemia [8].

Blood or tissue $n-3$ fatty acids have been reported to be associated with a lower risk of $\mathrm{CHD}[9,10]$. These results support the belief that measurements of circulating $n-3$ fatty acids may be useful for the prediction and management of cardiovascular risk. However, the benefits of measuring circulating fatty acids in people with type 2 diabetes in predicting the risks of cardiovascular and other vascular diseases are unclear. In addition, most studies report the results for individual fatty acids, and there are limited studies investigating multiple circulating fatty acids together.

To address these questions, we assessed the association of baseline plasma fatty acids with the risk of macrovascular and microvascular disease and death in participants with type 2 diabetes included in the Action in Diabetes and Vascular Disease: Preterax and Diamicron Modified-Release Controlled Evaluation (ADVANCE) study.

\section{Methods}

Participants and study design We performed a biomarker study using a case-cohort assessing the relationship between baseline plasma fatty acids and macrovascular and microvascular events and death in individuals with type 2 diabetes who participated in the ADVANCE trial (ClinicalTrials.gov registration no. NCT00145925). Between June 2001 and March 2003, 11,140 participants with type 2 diabetes were recruited for the ADVANCE trial, from 215 collaborating centres in 20 countries in Asia, Australasia, Europe and North America.

Individuals were potentially eligible for the ADVANCE trial if they had been diagnosed with type 2 diabetes mellitus at the age of 30 years or older and were aged 55 years or older at entry to the study. Potentially eligible participants also needed to have at least one of the following: a history of major CVD (stroke, myocardial infarction, hospital admission for transient ischaemic attack, hospital admission for unstable angina, coronary revascularisation, peripheral revascularisation, or amputation secondary to vascular disease) or at least one other risk factor for CVD. Such risk factors were defined by the presence of at least one of the following: a history of major microvascular disease (macroalbuminuria [urinary albumin/creatinine ratio $>300 \mu \mathrm{g} / \mathrm{mg}$ ], proliferative diabetic retinopathy, retinal photocoagulation therapy, macular oedema, or blindness in one eye thought to be caused by diabetes), current cigarette smoking, total cholesterol $>6.0 \mathrm{mmol} / 1$, HDL-cholesterol $<1.0 \mathrm{mmol} / 1$, microalbuminuria (urinary albumin/creatinine ratio 30-300 $\mu \mathrm{g}$ / $\mathrm{mg}$ ), diagnosis of type 2 diabetes mellitus made $\geq 10$ years before entry, or age $\geq 65$ years at entry. The trial included two randomised interventions: (1) a double-blind assessment of the efficacy of perindopril/indapamide ( $2 \mathrm{mg} / 0.625 \mathrm{mg}$ for $3 \mathrm{months}$, increasing to $4 \mathrm{mg} / 1.25 \mathrm{mg}$ if tolerated) vs placebo; and (2) an open-label evaluation of an intensive glucose-lowering regimen using modified-release gliclazide (with a target $\mathrm{HbA}_{1 \mathrm{c}} \leq$ $48 \mathrm{mmol} / \mathrm{mol}$ [6.5\%]) vs standard care.

For the present biomarker study, blood samples were available from all countries participating in the ADVANCE trial, except China and India, giving a base population of 7376 (Fig. 1). We included 4197 individuals from the case-cohort study, who comprised a random subcohort of 3500 individuals (2860 'controls' and 640 'cases') that was enriched with 697 additional 'cases' with a macrovascular or microvascular event or who had died during follow-up but were not in the subcohort. Full details of the study design and primary endpoints of the ADVANCE trial $[11,12]$ and its case-cohort [13] have been published elsewhere.

Proton NMR analysis Plasma samples were obtained at baseline from all study participants when they were in an unfasted state, given that these were people with type 2 diabetes at risk of hypoglycaemic episodes. Samples were collected across sites in a pragmatic fashion (commensurate with a multinational RCT) according to local facilities. Plasma samples were separated and stored centrally at $-80^{\circ} \mathrm{C}$ until measurement. The present study used a previously unthawed aliquot of plasma for proton NMR ( ${ }^{1} \mathrm{H}-\mathrm{NMR}$ ) analysis. ${ }^{1} \mathrm{H}-\mathrm{NMR}$ spectroscopy was performed on all available EDTA plasma samples from the ADVANCE casecohort study at baseline using a low-volume $(100 \mu \mathrm{l})$ variation of the quantitative ${ }^{1} \mathrm{H}-\mathrm{NMR}$ method (Nightingale Health, Helsinki, Finland) described previously [14, 15] and reviewed [16]. Sample spectra were analysed on a Bruker AVANCE III HD spectrometer (Billerica, MA, USA) to quantify a targeted list of metabolites, lipids and lipoproteins, as described previously [16].

This study presents new data on all fatty acids that can be robustly quantify by NMR, i.e. two individual fatty acids: DHA (an n-3 fatty acid) and linoleic acid (LA; an $n-6$ fatty acid). Six aggregate measures included: $n-3, n-6$, polyunsaturated (PUFA; sum of $n-3$ and $n-6$ fatty acids), monounsaturated (MUFA) and 
Fig. 1 Flow diagram for design of ADVANCE case-cohort study of fatty acid biomarkers for macrovascular events, microvascular events and death. ${ }^{\mathrm{a}}$ Macrovascular events, microvascular events and death are not mutually exclusive

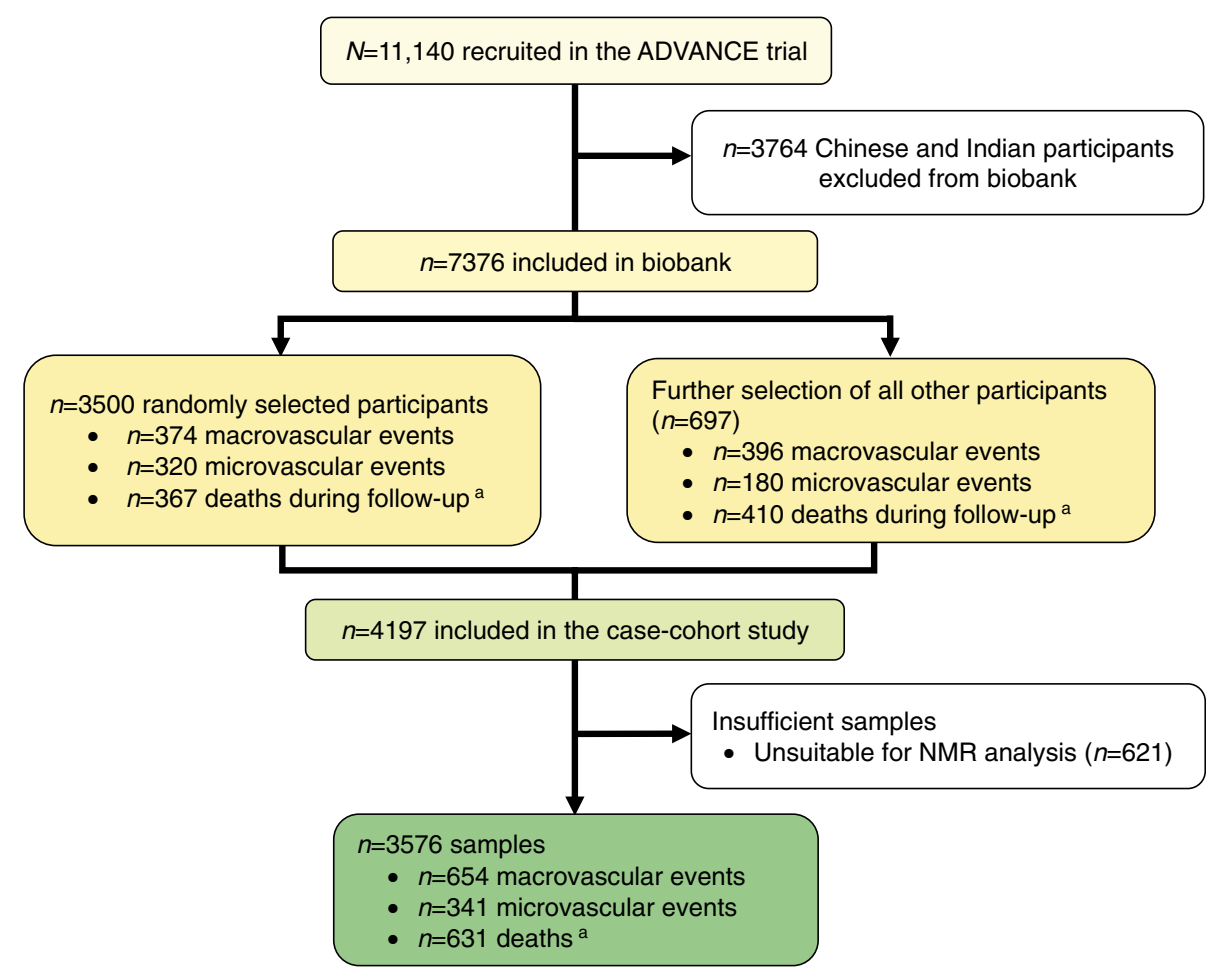

saturated (SFA) fatty acids. The percentages of total fatty acids as well as the absolute levels were used as the exposures of interest for the current analysis.

Study outcomes The primary outcomes for this study were major macrovascular and microvascular events and death that occurred during a median of 5 years of follow-up. An independent endpoint adjudication committee validated all these outcomes using ICD codes (electronic supplementary material [ESM] Methods). Major macrovascular events were cardiovascular death, non-fatal myocardial infarction and non-fatal stroke. Major microvascular events were a composite of new or worsening nephropathy or retinopathy, defined as any of the following: (1) development of macroalbuminuria; (2) doubling of serum creatinine level to $\geq 200 \mu \mathrm{mol} / 1$; (3) the need for renal replacement therapy due to kidney disease, or death due to renal disease; (4) development of proliferative retinopathy; (5) development of macular oedema; (6) occurrence of diabetes-related blindness; (7) use of retinal photocoagulation therapy. The secondary outcomes were individual components of macrovascular and microvascular events: cardiovascular death, non-fatal stroke, non-fatal myocardial infarction and new or worsening nephropathy.

Statistical analyses Baseline participant characteristics were summarised according to the study outcomes (major macrovascular and microvascular events and death). Categorical data were presented as number (percentage), and continuous data according to the data distribution, mean (SD) for approximately symmetrically distributed data and median (interquartile interval [IQI]) for skewed distributions. The percentage contribution of the fatty acid biomarkers of the total fatty acid and the absolute fatty acid values were summarised as mean (SD) values for each study outcome.

Cox proportional hazards models for case-cohort data were used to model the associations between fatty acids and the study outcomes, adjusting covariates considered as traditional cardiovascular risk factors. Models estimated HR (per SD percentage higher) of the percentage contribution of total fatty acids. Two sets of models were fit for each fatty acid-outcome combination: model 1, adjusted for age, sex, region and the treatments randomly allocated in the RCT; and multiple-adjusted model 2, additionally adjusted for history of macrovascular disease, duration of diabetes, current smoking status, systolic BP, BMI, urinary albumin/creatinine ratio, eGFR (calculated using the Chronic Kidney Disease Epidemiology Collaboration [CKD-EPI] creatinine equation), $\mathrm{HbA}_{1 \mathrm{c}}$, HDL-cholesterol, triacylglycerols, and use of aspirin or other antiplatelet agents, statins or other lipidlowering agents, $\beta$-blockers and ACE inhibitors or angiotensin receptor blockers. All $p$ values reported are two-sided, with the $5 \%$ threshold used to determine statistical significance. Since several statistical tests are included in this article, the reader is recommended to treat marginal levels of significance with caution.

Sensitivity analyses included models estimating HRs for 1 SD higher of the absolute level of each fatty acid. For the fatty acid-outcome combinations that yielded statistically significant results in multiple-adjusted models in the main analysis, models were fitted estimating HRs in quarters of the range of values of the percentage of fatty acids. 
Subgroup analyses were performed according to baseline covariates, including age ( $<65$ years or $\geq 65$ years), sex, region of residence (Australia, New Zealand and South East Asia, Canada, Continental Europe and Northern Europe), history of macrovascular disease, history of microvascular disease, eGFR $\left(<60 \mathrm{ml} \mathrm{min}^{-1}[1.73 \mathrm{~m}]^{-2}\right.$ or $\left.\geq 60 \mathrm{ml} \mathrm{min}^{-1}[1.73 \mathrm{~m}]^{-2}\right)$, triacylglycerols $(<1.7 \mathrm{mmol} / 1$ or $\geq 1.7 \mathrm{mmol} / \mathrm{l})$, and randomised treatments (BP- and glucose-lowering treatments).

The ability of fatty acids to discriminate between those who will and those who will not go on to suffer major macrovascular and microvascular events and death was estimated using $\mathrm{C}$ statistics accounting for censoring $[17,18]$. Further, the ability of fatty acids to reclassify participants was estimated using the continuous net reclassification improvement (NRI) [19, 20]. These statistics were computed, for 5 year risk, for individuals in the random subcohort only for those fatty acids whose percentage contribution demonstrated a statistically significant association with outcomes. 95\% CIs for the C statistic (and increments in it when adding fatty acids) and NRI were calculated using bootstrap methods with a normal approximation and 500 bootstrap

Table 1 Baseline characteristics of participants in the case-cohort study by macrovascular events, microvascular events and death

\begin{tabular}{|c|c|c|c|c|c|c|}
\hline \multirow[t]{2}{*}{ Characteristic } & \multicolumn{2}{|c|}{ Macrovascular events } & \multicolumn{2}{|c|}{ Microvascular events } & \multicolumn{2}{|l|}{ Death } \\
\hline & Yes & No & Yes & No & Yes & No \\
\hline$N(\%)$ & $654(18.3)$ & $2922(81.7)$ & $341(9.5)$ & $3235(90.5)$ & $631(17.6)$ & $2945(82.4)$ \\
\hline Age, years & $69(7)$ & $66(7)$ & $66(6)$ & $67(7)$ & $70(7)$ & $66(6)$ \\
\hline Men, $n(\%)$ & $450(69)$ & $1712(59)$ & $226(66)$ & $1936(60)$ & $438(69)$ & $1724(59)$ \\
\hline \multicolumn{7}{|l|}{ Region, $n(\%)$} \\
\hline ANZ/SEA & $155(24)$ & $713(24)$ & $123(36)$ & $745(23)$ & $120(19)$ & $748(25)$ \\
\hline Canada & $33(5)$ & $185(6)$ & $28(8)$ & $190(6)$ & $34(5)$ & $184(6)$ \\
\hline Continental Europe & $262(40)$ & $1154(39)$ & $90(26)$ & $1326(41)$ & $264(42)$ & $1152(39)$ \\
\hline Northern Europe & $204(31)$ & $870(30)$ & $100(29)$ & $974(30)$ & $213(34)$ & $861(29)$ \\
\hline Duration of diabetes, years & $9.2(7.1)$ & $7.6(6.3)$ & $9.8(6.9)$ & $7.7(6.4)$ & $9.2(7.6)$ & $7.6(6.2)$ \\
\hline History of macrovascular disease, $n(\%)$ & $323(49)$ & $925(32)$ & $118(35)$ & $1130(35)$ & $283(45)$ & $965(33)$ \\
\hline Current smoker, $n(\%)$ & $84(13)$ & $390(13)$ & $44(13)$ & $430(13)$ & $96(15)$ & $378(13)$ \\
\hline Systolic BP, mmHg & $150(23)$ & $146(21)$ & $150(21)$ & $147(22)$ & $149(23)$ & $147(21)$ \\
\hline Diastolic BP, mmHg & $82(11)$ & $82(11)$ & $82(11)$ & $82(11)$ & $81(12)$ & $82(11)$ \\
\hline $\mathrm{HbA}_{1 \mathrm{c}}, \mathrm{mmol} / \mathrm{mol}$ & $60(17)$ & $57(15)$ & $61(18)$ & $57(15)$ & $59(17)$ & $57(15)$ \\
\hline $\mathrm{HbA}_{1 \mathrm{c}}, \%$ & $7.6(1.6)$ & $7.4(1.4)$ & $7.8(1.6)$ & $7.4(1.4)$ & $7.6(1.6)$ & $7.4(1.4)$ \\
\hline eGFR, $\mathrm{ml} \mathrm{min}^{-1}(1.73 \mathrm{~m})^{-2}$ & $68(18)$ & $73(16)$ & $70(19)$ & $72(16)$ & $67(18)$ & $73(16)$ \\
\hline Urinary ACR, $\mu \mathrm{g} / \mathrm{mg}$ & $21(9,71)$ & $13(6,35)$ & $49(14,127)$ & $13(6,34)$ & $21(8,66)$ & $13(6,35)$ \\
\hline Total cholesterol, $\mathrm{mmol} / \mathrm{mol}$ & $5.1(1.2)$ & $5.2(1.2)$ & $5.2(1.1)$ & $5.1(1.2)$ & $5.1(1.1)$ & $5.2(1.2)$ \\
\hline HDL-cholesterol, mmol/mol & $1.17(0.31)$ & $1.23(0.33)$ & $1.18(0.31)$ & $1.23(0.33)$ & $1.18(0.31)$ & $1.23(0.33)$ \\
\hline Triacylglycerols, mmol/1 & $1.6(1.2,2.3)$ & $1.7(1.2,2.4)$ & $1.8(1.3,2.6)$ & $1.7(1.2,2.3)$ & $1.6(1.22 .3)$ & $1.7(1.22 .4)$ \\
\hline Randomised BP-lowering treatment, $n(\%)$ & $310(47)$ & $1453(50)$ & $163(48)$ & $1600(49)$ & $296(47)$ & $1467(50)$ \\
\hline Randomised intensive blood glucose control, $n(\%)$ & $321(49)$ & $1445(49)$ & $151(44)$ & $1615(50)$ & $309(49)$ & 1457 (49) \\
\hline \multicolumn{7}{|l|}{ Medication use, $n(\%)$} \\
\hline Aspirin or other antiplatelet agent & $386(59)$ & $1373(47)$ & $170(50)$ & $1589(49)$ & $351(56)$ & $1408(48)$ \\
\hline Statins or other lipid-lowering agent & $283(43)$ & $1305(45)$ & $157(46)$ & $1431(44)$ & $260(41)$ & $1328(45)$ \\
\hline$\beta$-blocker & $211(32)$ & $875(30)$ & $95(28)$ & $991(31)$ & $196(31)$ & $890(30)$ \\
\hline ACE inhibitor or angiotensin receptor blocker & $417(64)$ & $1664(57)$ & $231(68)$ & $1850(57)$ & $394(62)$ & $1687(57)$ \\
\hline \multicolumn{7}{|l|}{ Fatty acids, $\%$ of total fatty acids } \\
\hline PUFA & $28.8(5.8)$ & $29.0(5.7)$ & $28.2(5.8)$ & $29.1(5.7)$ & $28.9(5.5)$ & $29.0(5.8)$ \\
\hline$n$-3 Fatty acids & $2.5(1.3)$ & $2.8(1.4)$ & $2.7(1.4)$ & $2.7(1.4)$ & $2.6(1.3)$ & $2.8(1.4)$ \\
\hline DHA & $0.75(0.46)$ & $0.83(0.50)$ & $0.79(0.53)$ & $0.82(0.49)$ & $0.76(0.48)$ & $0.83(0.50)$ \\
\hline$n-6$ Fatty acids & $26.2(4.9)$ & $26.2(4.8)$ & $25.5(5.2)$ & $26.3(4.8)$ & $26.3(4.6)$ & $26.2(4.9)$ \\
\hline LA & $17.2(6.7)$ & $17.2(6.2)$ & $16.6(6.7)$ & $17.3(6.3)$ & $17.3(6.3)$ & $17.2(6.3)$ \\
\hline MUFA & $30.2(3.6)$ & $30.2(3.7)$ & $30.4(5.4)$ & $30.2(3.5)$ & $30.2(3.7)$ & $30.2(3.7)$ \\
\hline SFA & $41.0(5.1)$ & $40.8(5.0)$ & $41.6(5.9)$ & $40.8(5.0)$ & $40.9(5.0)$ & $40.8(5.1)$ \\
\hline \multicolumn{7}{|l|}{ Fatty acids, mmol/l } \\
\hline Total fatty acids & $8.34(2.91)$ & $8.63(3.18)$ & $8.50(2.93)$ & $8.59(3.16)$ & $8.31(2.61)$ & $8.64(3.24)$ \\
\hline PUFA & $2.45(1.01)$ & $2.55(1.03)$ & $2.45(1.03)$ & $2.53(1.03)$ & $2.43(0.92)$ & $2.55(1.05)$ \\
\hline$n-3$ Fatty acids & $0.23(0.15)$ & $0.26(0.17)$ & $0.24(0.17)$ & $0.25(0.17)$ & $0.23(0.15)$ & $0.26(0.17)$ \\
\hline DHA & $0.067(0.049)$ & $0.077(0.056)$ & $0.073(0.057)$ & $0.076(0.055)$ & $0.068(0.050)$ & $0.077(0.056)$ \\
\hline$n-6$ Fatty acids & $2.21(0.88)$ & $2.29(0.89)$ & $2.21(0.89)$ & $2.28(0.88)$ & $2.20(0.79)$ & $2.29(0.90)$ \\
\hline LA & $1.52(0.84)$ & $1.56(0.83)$ & $1.50(0.83)$ & $1.56(0.83)$ & $1.51(0.75)$ & $1.57(0.84)$ \\
\hline MUFA & $2.55(1.05)$ & $2.64(1.16)$ & $2.61(1.07)$ & $3.43(1.15)$ & $2.54(0.95)$ & $2.64(1.17)$ \\
\hline SFA & $3.34(1.06)$ & $3.46(1.23)$ & $3.45(1.08)$ & $3.43(1.22)$ & $3.33(0.95)$ & $3.46(1.25)$ \\
\hline
\end{tabular}

Data are presented as mean (SD) or median with IQI (lower quartile, upper quartile), unless otherwise stated ACR, albumin/creatinine ratio; ANZ/SEA, Australia and New Zealand/South-East Asia; IQI, interquartile interval 
iterations. All analyses in this study were performed using Stata/ MP, version 15 (Stata Corporation, College Station, TX, USA), and R, version 3.5.3 (R Foundation for Statistical Computing, Vienna, Austria); the R code is provided in the ESM Methods.

\section{Results}

Participants' characteristics There were 3576 individuals from the case-cohort that had available data for at least one fatty acid, which comprised 3021 from the random subcohort (2507 controls and 514 cases) and 555 additional cases. For the 3576 individuals there were 654 macrovascular events, 341 microvascular events and 631 deaths during a median of 5 years of follow-up (Fig. 1).

Higher percentages of the fatty acids of total fatty acids in those free from an adverse event were observed, compared with lower percentages in those with adverse events. Significant differences were observed in the percentage of $n-3$ fatty acids and DHA for macrovascular events and death, and in PUFA, SFA, $n-6$ fatty acids and LA in microvascular events (Table 1). Similar findings were observed in the absolute fatty acid levels, for most fatty acids considered in this study, where the mean levels were significantly higher in participants who were free from major macrovascular events and alive at the end of the study. There were no significant differences in the absolute level of any fatty acids for microvascular events.

Clinical outcomes during follow-up After adjustment for age, sex, region and randomised treatments (model 1), there were highly significant inverse associations with the risk of macrovascular events and death for the percentage of $n-3$ fatty acids (HR [95\% CI]: 0.84 [0.77, 0.91] and 0.85 [0.79, 0.93], respectively, per $1 \mathrm{SD}$ higher percentage) and DHA (HR [95\% $\mathrm{CI}]: 0.82$ [0.76, 0.89]) and $0.85[0.78,0.92]$, respectively, per 1 SD higher percentage) (ESM Fig. 1). After further adjustment (model 2), the association remained, albeit weaker, for the percentage of $n-3$ fatty acids (HR [95\% CI]: 0.87 [0.80, 0.95] and $0.91[0.84,0.99]$ for the risk of macrovascular events and death, respectively, per 1 SD higher percentage) and for DHA (HR [95\% CI]: 0.88 [0.81, 0.96] and 0.93 [0.85, 1.01] for the risk of macrovascular events and death, respectively, per 1 SD higher percentage), although the latter was not significant (Fig. 2).

For individual components of macrovascular events from multiple-adjusted models, $n$-3 fatty acids and DHA were associated with lower risks of cardiovascular death (HR [95\% CI]: 0.85 $[0.75,0.96]$ and $0.86[0.76,0.98])$, respectively, and non-fatal stroke (HR [95\%CI]: $0.82[0.69,0.97]$ and 0.82 [0.69, 0.97]),

\begin{tabular}{|c|c|}
\hline & $\mathrm{HR}(95 \% \mathrm{Cl})$ \\
\hline \multicolumn{2}{|l|}{ Macrovascular events } \\
\hline PUFA $(1 S D=5.8)$ & $0.94(0.86,1.03)$ \\
\hline$n-3$ Fatty acids $(1 \mathrm{SD}=1.4)$ & $0.87(0.80,0.95)$ \\
\hline $\mathrm{DHA}(1 \mathrm{SD}=0.49)$ & $0.88(0.81,0.96)$ \\
\hline$n-6$ Fatty acids $(1 S D=4.8)$ & $0.97(0.89,1.07)$ \\
\hline $\mathrm{LA}(1 \mathrm{SD}=6.3)$ & $0.98(0.90,1.07)$ \\
\hline MUFA $(1 S D=3.7)$ & $1.07(0.96,1.19)$ \\
\hline $\mathrm{SFA}(1 \mathrm{SD}=5.1)$ & $1.03(0.95,1.11)$ \\
\hline \multicolumn{2}{|l|}{ Microvascular events } \\
\hline PUFA $(1 S D=5.8)$ & $0.98(0.87,1.11)$ \\
\hline$n-3$ Fatty acids $(1 \mathrm{SD}=1.4)$ & $1.01(0.91,1.13)$ \\
\hline $\mathrm{DHA}(1 \mathrm{SD}=0.49)$ & $1.01(0.91,1.13)$ \\
\hline$n-6$ Fatty acids $(1 \mathrm{SD}=4.8)$ & $0.97(0.86,1.10)$ \\
\hline $\mathrm{LA}(1 \mathrm{SD}=6.3)$ & $0.98(0.87,1.10)$ \\
\hline MUFA $(1 S D=3.7)$ & $0.97(0.85,1.12)$ \\
\hline $\mathrm{SFA}(1 \mathrm{SD}=5.1)$ & $1.07(0.96,1.19)$ \\
\hline \multicolumn{2}{|l|}{ Death } \\
\hline PUFA $(1 S D=5.8)$ & $0.95(0.86,1.04)$ \\
\hline$n-3$ Fatty acids $(1 S D=1.4)$ & $0.91(0.84,0.99)$ \\
\hline $\mathrm{DHA}(1 \mathrm{SD}=0.49)$ & $0.93(0.85,1.01)$ \\
\hline$n-6$ Fatty acids $(1 S D=4.8)$ & $0.97(0.88,1.07)$ \\
\hline $\operatorname{LA}(1 \mathrm{SD}=6.3)$ & $0.98(0.85,1.07)$ \\
\hline MUFA $(1 \mathrm{SD}=3.7)$ & $1.03(0.92,1.15)$ \\
\hline $\mathrm{SFA}(1 \mathrm{SD}=5.1)$ & $1.04(0.95,1.13)$ \\
\hline
\end{tabular}

Fig. 2 Adjusted HRs for macrovascular events, microvascular events and death associated with fatty acid levels (per $1 \mathrm{SD}$ increase in percentage of total fatty acids), using multiple-adjusted models. Models were adjusted for age, sex, region, randomised treatment, history of macrovascular disease, duration of diabetes, current smoking status, systolic BP, BMI, urinary albumin/creatinine ratio, eGFR, $\mathrm{HbA}_{1 \mathrm{c}}$, HDL-cholesterol, triacylglycerols, and use of aspirin or other antiplatelet agents, statins or other lipid-lowering agents, $\beta$-blockers, and ACE inhibitors or angiotensin receptor blockers 


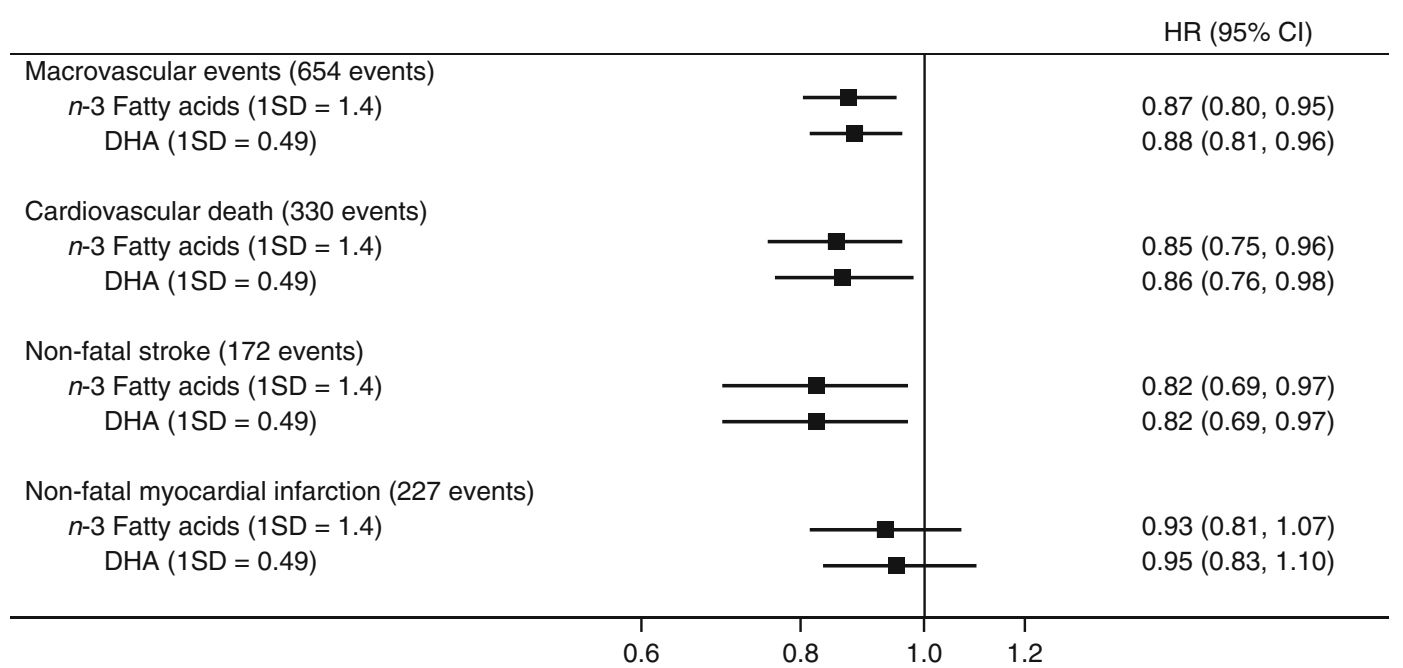

Fig. 3 Adjusted HRs for individual components of macrovascular events (cardiovascular death, non-fatal myocardial infarction, non-fatal stroke) associated with $n-3$ fatty acid and DHA levels (per 1 SD increase in percentage of total fatty acids,) using multiple-adjusted models. Models were adjusted for age, sex, region, randomised treatment, history of

respectively (Fig. 3). The statistically significant associations did not hold for non-fatal myocardial infarction.

Further, significant inverse associations were suggested after minor adjustment in the percentage of PUFA on macrovascular events, and in PUFA, $n-6$ fatty acids and LA on the risk of death (ESM Fig. 1), but these findings did not persist after further adjustment (Fig. 2). No significant associations were observed between any fatty acids and the risk of microvascular events (Fig. 2) as well as new or worsening nephropathy (ESM Fig. 2).

Further analyses of quarters of fatty acids levels revealed that the inverse associations with the percentage of $n-3$ and DHA were approximately linear with the adjusted HRs for macrovascular events and death (ESM Fig. 3). The associations in the absolute values of the fatty acids were in line with the percentage fatty acid, albeit weaker (ESM Fig. 4).

Subgroup analyses for $n-3$ fatty acids and DHA Subgroup analyses were undertaken for $n-3$ fatty acids and DHA with macrovascular events and death (ESM Figs 5 and 6). There were no significant interactions between any subgroups ( $p$ for interaction $>0.1$ ) other than triacylglycerols $(p$ for interaction $=0.02$ ) and randomised BP-lowering treatment $(p$ for interaction $=$ 0.01 ) for $n-3$ fatty acids and the risk of macrovascular events. A further subgroup analysis (ESM Table 1) for major microvascular outcomes in those free from microvascular disease at baseline yielded HRs [95\% CI] for $n-3$ of $0.94[0.82,1.08]$ and for DHA of $0.94[0.82,1.08]$. The $p$ value for interaction indicates that there was no statistically significant interaction in the association of $n-3(p=0.243)$ or DHA $(p=0.247)$ and major microvascular outcomes by history of microvascular disease at baseline. macrovascular disease, duration of diabetes, current smoking status, systolic BP, BMI, urinary albumin/creatinine ratio, eGFR, $\mathrm{Hb}_{1 \mathrm{c}}$, HDLcholesterol, triacylglycerols, and use of aspirin or other antiplatelet agents, statins or other lipid-lowering agents, $\beta$-blockers, ACE inhibitors or angiotensin receptor blockers

Prognostic value of fatty acids compared with traditional risk factors The difference in the $\mathrm{C}$ statistics between the base model with age, sex, region and randomised treatments without fatty acids and the model which included the index fatty acids demonstrated small improvements for predicting macrovascular events for DHA (difference: 0.0104 [95\% CI $0.0001,0.0206]$ ) and for predicting death for $n-3$ fatty acids (difference: 0.0103 [95\% CI 0.0004, 0.0202]) and DHA (difference: 0.0084 [95\% CI 0.0000, 0.0169]) (ESM Table 2). There were no statistically significant differences in the $\mathrm{C}$ statistics for other fatty acids. The inclusion of $n-3$ fatty acids and DHA, in comparison with base model 1 , yielded the largest improvements in the continuous NRI, although not statistically significant: 0.157 (95\% CI -0.006 , $0.267)$ and $0.156(95 \% \mathrm{CI}-0.009,0.265)$, respectively, for macrovascular events; and 0.170 (95\% CI $-0.013,0.293)$ and 0.161 (95\% CI $-0.008,0.272)$, respectively, for death (ESM Table 2). After including fatty acids in model 2, which included many traditional cardiovascular risk factors, there were limited improvements in the $\mathrm{C}$ statistic and continuous NRI for $n$-3 fatty acids and DHA, although there were no longer any significant associations (Table 2).

\section{Discussion}

This biomarker study showed inverse associations of baseline plasma $n-3$ fatty acids and DHA with the risk of macrovascular events and for $n-3$ fatty acids with the risk of death among individuals with type 2 diabetes. These inverse associations appeared approximately linear, and among the macrovascular events, $n-3$ fatty acids and DHA demonstrated 
Table 2 Prognostic value of fatty acids, compared with traditional CVD risk factors, using $\mathrm{C}$ statistic (and difference) and continuous NRI, with $95 \%$ CIs, for macrovascular events and death (per $1 \mathrm{SD}$ increase in percentage of total fatty acids): results from multiple-adjusted models

\begin{tabular}{|c|c|c|}
\hline & $\mathrm{C}$ statistic and difference $(95 \% \mathrm{CI})^{\mathrm{a}}$ & Continuous NRI $(95 \% \mathrm{CI})$ \\
\hline \multicolumn{3}{|l|}{ Macrovascular events } \\
\hline Multiple-adjusted model $2^{\mathrm{b}}$ & $0.6919(0.6467,0.7101)$ & - \\
\hline$+n$-3 Fatty acids & $+0.0034(-0.0035,0.0102)$ & $0.144(-0.019,0.263)$ \\
\hline + DHA & $+0.0025(-0.0027,0.0076)$ & $0.121(-0.066,0.254)$ \\
\hline \multicolumn{3}{|l|}{ Death } \\
\hline Multiple-adjusted model $2^{\mathrm{b}}$ & $0.7044(0.6641,0.7220)$ & - \\
\hline$+n-3$ Fatty acids & $+0.0057(-0.0005,0.0119)$ & $0.124(-0.047,0.257)$ \\
\hline + DHA & $+0.0042(-0.0004,0.0087)$ & $0.145(-0.070,0.274)$ \\
\hline
\end{tabular}

stronger associations with cardiovascular death and non-fatal stroke. In contrast, no significant associations were observed for the predominant fatty acids such as $n-6$ fatty acids, LA, MUFA and SFA with the risk of macrovascular events and death, after adjustment for multiple traditional risk factors.

The prospective associations of circulating $n-3$ fatty acids and DHA with the risk of CVD are consistent with the prior studies. According to previous pooled analyses, DHA in whole plasma was associated with a lower risk of fatal and non-fatal CHD (RR [95\% CI]: 0.78 [0.69, 0.90] and 0.91 $[0.84,0.98]$, respectively) in people without a history of CVD [9]. In addition, another study reported that plasma $n-3$ fatty acids were associated with a lower risk of non-fatal myocardial infarction [21]. Similar inverse associations with CHD and stroke were observed in other studies which measured $n-3$ fatty acids in plasma phospholipid [ 10 , 22-24], whole blood [25] and serum [26], among people without prior CVD. These studies did not assess the C statistic and NRI; however, the present study did not detect significant improvement in predicting macrovascular events by adding $n-3$ fatty acids or DHA into the model including traditional cardiovascular risk factors. This indicates that the predictive power of plasma $n$-3 fatty acids and DHA may be limited in the presence of a complement of traditional cardiovascular risk factors. On the other hand, cardiovascular benefits of high-dose supplementation with $n-3$ fatty acids were recently observed in REDUCE-IT, where there were potentially greater benefits of $n$ - 3 fatty acid supplementation in those with lower plasma levels of $n-3$ fatty acids.

In contrast to generally consistent results from observational studies of circulating $n-3$ fatty acids and DHA, the effects of $n-3$ fatty acid supplementation on cardiovascular outcomes in RCTs have been mixed [4, 6, 27-30]. The recent study, A Study of Cardiovascular Events in Diabetes (ASCEND) trial, of 15,480 individuals with diabetes free of prior CVD, which tested $n-3$ fatty acid supplementation ( $1 \mathrm{~g} /$ day) for 7.4 years, did not lower the risk of composite major vascular outcomes, while only vascular deaths were less frequent in the supplementation group than in the placebo group (RR 0.82 [95\% CI $0.68,0.98]$ ] [31]. On the other hand, the recent AHA science advisory has suggested that the use of $n-3$ fatty acid supplementation was probably justified in individuals at high cardiovascular risk [8, 28]. In REDUCE-IT, which used high-dose (4 g/day) n-3 fatty acid supplementation in 8179 individuals with established CVD or with diabetes and other risk factors, the risk of composite cardiovascular outcomes was substantially reduced (HR 0.75 [95\% CI 0.68, 0.83]) [7]. In addition, some secondary analyses from large trials have reported the benefit of $n-3$ fatty acid supplementation on CVD in diabetic populations with hypercholesterolaemia [32], chronic heart failure [33] and history of myocardial infarction [34]. Further investigation of the benefits of $n-3$ fatty acid supplementation will, therefore, be required among people with type 2 diabetes and high CVD risks.

Several mechanisms may explain the favourable associations between $n-3$ fatty acids and the risk of CVD. Previous clinical trials looking at intermediate cardiovascular outcomes among people with diabetes have reported that $n$-3 fatty acid supplementation could lower triacylglycerol concentrations $[35,36]$, improve arterial blood flow and attenuate inflammatory signals [37, 38]. These effects were supported by clinical trials in the general population and in experimental studies [39-41].

The present study observed the inverse associations between baseline plasma $n-6$ fatty acids and LA with the risk of death, but these associations attenuated after adjustment for multiple risk factors. Similar findings have been reported between serum LA and mortality in a cohort of older adults ( $\geq 65$ years of age) [42]. We did not, however, detect significant associations between $n-6$ fatty acids and LA with the risk 
of macrovascular events. These results were consistent with those of previous observational studies [23, 24, 43] and directionally concordant with the recent pooled analyses of 30 cohort studies which reported that higher circulating and tissue levels of LA were associated with a lower risk of major cardiovascular events (HR 0.93 [95\% CI 0.88, 0.99]) [44]. In addition, our study did not detect significant associations with the risk of CVD for circulating MUFA and SFA, which is consistent with previous studies [24, 43, 45], while some studies have reported that MUFA in blood were associated with a higher risk of CVD [46].

There were no significant associations between any fatty acids and the risk of microvascular events. A further analysis specifically for renal outcome also demonstrated no significant associations. Although limited studies have assessed the association between circulating fatty acids and renal outcomes, in an Italian cohort of 931 adults, plasma $n-3$ fatty acids were inversely associated with the risk of developing renal insufficiency (creatinine clearance rate $<60 \mathrm{ml} / \mathrm{min}$ ) [47]. However, in a cohort of 2792 individuals, levels of $n-3$ and $n-6$ fatty acids and SFA in plasma phospholipid were not associated with kidney function [48].

The strengths of the current study include the use of an efficiently designed case-cohort study from a wellcharacterised clinical trial to yield a powerful study for a range of outcomes, which were independently adjudicated according to pre-specified criteria. This study included multiple plasma fatty acids and the ability to adjust for multiple covariates including lipids and lipid-lowering drugs, such as statins. We also considered the percentage that individual fatty acids contributed to total fatty acids, as well as the absolute levels of fatty acid, and both measures are important for interpreting fatty acid values since an increased intake of a specific fatty acid could alter the relative percentage of other fatty acids while their absolute levels are unlikely to be altered. The present study, however, has several limitations. First, as the study cohort was derived from a randomised trial of individuals with type 2 diabetes, our results may have limited generalisability to broader populations. Second, as fatty acids were measured in pragmatically collected plasma samples in a randomised trial, we cannot rule out the potential for differential preanalytical sample handling or sample degradation during storage, which may have biased our results [49]. Further, as plasma samples were collected from non-fasted participants, the levels of fatty acids might have been affected by the consumption of a recent meal [50], although, in clinical practice, fasting is rarely required among individuals with type 2 diabetes. Third, fatty acids were only measured in plasma samples collected at study baseline; thus, we were unable to consider how the change in fatty acid values during the study follow-up might have influenced the exposure-outcome association. Finally, our study considered only two individual fatty acids (DHA and LA), since the resolution of the employed high- throughput NMR platform was limited in terms of individual fatty acid types, and only allowed robust quantification of DHA within $n-3$, and LA within $n-6$. The specific set of measures was determined by their overall concentration in plasma and also on spectroscopic aspects, such as overlapping signals, which makes it challenging to quantify from native plasma where no lipid extraction is used [14]. LA and DHA were reported since they generate distinct peaks in the spectral data produced by the measurement, and we were able quantify them separately as part of our high-throughput service. However, fatty acid concentrations quantified by the NMR metabolomics platform were highly consistent with the concentrations compared with GC, the latter being challenging with large samples [46]. Further, NMR, is a novel technology with the potential of offering a cost-effective platform for multiple biomarker testing and has great potential in regard to fatty acid measurement.

In conclusion, we report distinct associations of different plasma fatty acids with the risk of major clinical outcomes in individuals with type 2 diabetes. In particular, plasma $n-3$ fatty acids were associated with a lower risk of macrovascular disease and death, and DHA was associated with a lower risk of macrovascular disease. These results support the cardioprotective effects of $n-3$ fatty acids and DHA and further merit testing the role of high-dose $n-3$ fatty acid supplementation in individuals with type 2 diabetes.

Data availability Restrictions apply to the availability of these data, which were used by agreement of the ADVANCE steering committee for the current study, and so are not publicly available.

Funding The biomarker work in the present study was funded by the Chest Heart and Stroke Association Scotland (R13/A149) and by the Glasgow Molecular Pathology Node, which is funded by the Medical Research Council and The Engineering and Physical Sciences Research Council (MR/N005813/1). The ADVANCE trial (ClinicalTrials.gov registration no. NCT00145925) was funded by the National Health and Medical Research Council (NHMRC) of Australia (project grant ID 211086 and program grant IDs 358395 and 571281) and by Servier. MO is supported by the Japan Society for the Promotion of Science Program for Fostering Globally Talented Researchers. MJ is supported by a Scientia fellowship from UNSW Sydney. MW is an NHMRC of Australia principal research fellow (1080206). The study sponsors were not involved in the design of the study, the collection, analysis, and interpretation of data, writing the report or the decision to submit the report for publication.

Authors' relationships and activities MW reports consultancy fees from Amgen and Kirin and is supported by an Australian NHMRC fellowship (APP1080206) and MRC program grant (APP1149987). JC received research grants from the NHMRC of Australia and from Servier for the ADVANCE trial and ADVANCE-ON post-trial follow-up, and honoraria for speaking about these studies at scientific meetings; he also reports grant support from a program grant (APP1149987) from the NHMRC of Australia. MJ reports receiving grant support from the NHMRC of Australia (project grant: 1148060) and unrestricted grant support from VentureWise (a wholly owned commercial subsidiary of NPS MedicineWise) to conduct a commissioned project funded by AstraZeneca. PWü is an employee and shareholder of Nightingale 
Health Ltd., which conducted the biomarker quantification. NP has received personal speaker fees from Servier, Takeda and Novo Nordisk, and advisory board activities from Astra Zeneca and Novo Nordisk. He has also received grants for his research group relating to type 2 diabetes mellitus from Diabetes UK, NIHR EME, Julius Clinical and the British Heart Foundation. He holds no stocks and shares in any such company. All other authors declare that there is no duality of interest associated with their contribution to this manuscript.

Contribution statement MW and JC conceived, designed and acquired the ADVANCE trial data. PWe, MW and NS conceived this secondary study, and PWe and NS obtained grant funding. PWü acquired biomarker data and $\mathrm{KH}$ and $\mathrm{MO}$ undertook the statistical analyses, with advice from MW. All authors were involved in data interpretation. $\mathrm{KH}$ and $\mathrm{MO}$ wrote the initial drafts of the manuscript. These drafts were revised for important scientific content by all authors. All authors gave final approval of the version to be published. MW is the guarantor of this work.

Open Access This article is licensed under a Creative Commons Attribution 4.0 International License, which permits use, sharing, adaptation, distribution and reproduction in any medium or format, as long as you give appropriate credit to the original author(s) and the source, provide a link to the Creative Commons licence, and indicate if changes were made. The images or other third party material in this article are included in the article's Creative Commons licence, unless indicated otherwise in a credit line to the material. If material is not included in the article's Creative Commons licence and your intended use is not permitted by statutory regulation or exceeds the permitted use, you will need to obtain permission directly from the copyright holder. To view a copy of this licence, visit http://creativecommons.org/licenses/by/4.0/.

\section{References}

1. International Diabetes Federation (2017) IDF diabetes atlas. International Diabetes Federation, Brussels, Belgium

2. Ma J, Folsom AR, Shahar E, Eckfeldt JH (1995) Plasma fatty acid composition as an indicator of habitual dietary fat intake in middleaged adults. The Atherosclerosis Risk in Communities (ARIC) study investigators. Am J Clin Nut 62(3):564-571. https://doi.org/ 10.1093/ajen/62.3.564

3. Hafizi Abu Bakar M, Kian Kai C, Wan Hassan WN, Sarmidi MR, Yaakob H, Zaman Huri H (2015) Mitochondrial dysfunction as a central event for mechanisms underlying insulin resistance: the roles of long chain fatty acids. Diabetes Meta Res Rev 31(5):453475. https://doi.org/10.1002/dmrr.2601

4. Abdelhamid AS, Brown TJ, Brainard JS et al (2018) Omega-3 fatty acids for the primary and secondary prevention of cardiovascular disease. Cochrane Database Syst Rev (7):CD003177. https://doi. org/10.1002/14651858.CD003177.pub3

5. Kotwal S, Jun M, Sullivan D, Perkovic V, Neal B (2012) Omega 3 fatty acids and cardiovascular outcomes: systematic review and meta-analysis. Cir. Cardiovas Qual Outcomes 5(6):808-818. https://doi.org/10. 1161/CIRCOUTCOMES.112.966168

6. Hu Y, Hu FB, Manson JE (2019) Marine omega-3 supplementation and cardiovascular disease: an updated meta-analysis of 13 randomized controlled trials involving 127477 participants. J Am Heart Assoc 8(19):e013543. https://doi.org/10.1161/JAHA.119. 013543

7. Bhatt DL, Steg PG, Miller M et al (2019) Cardiovascular risk reduction with icosapent ethyl for hypertriglyceridemia. N Eng $\mathrm{J}$ of Med 380(1):11-22. https://doi.org/10.1056/NEJMoa1812792
8. Skulas-Ray AC, Wilson PWF, Harris WS et al (2019) Omega-3 fatty acids for the management of hypertriglyceridemia: a science advisory from the American Heart Association. Circulation 140(12): e673-e691. https://doi.org/10.1161/CIR.0000000000000709

9. Del Gobbo LC, Imamura F, Aslibekyan S et al (2016) $\omega-3$ polyunsaturated fatty acid biomarkers and coronary heart disease: pooling project of 19 cohort studies. JAMA Intern Med 176(8):1155-1166. https://doi.org/10.1001/jamainternmed.2016.2925

10. de Oliveira Otto MC, Wu JH, Baylin A et al (2013) Circulating and dietary omega- 3 and omega- 6 polyunsaturated fatty acids and incidence of CVD in the Multi-Ethnic Study of Atherosclerosis. J A Heart Assoc 2(6):e000506. https://doi.org/10.1161/JAHA.113. 000506

11. Patel A, MacMahon S, Chalmers J et al (2007) Effects of a fixed combination of perindopril and indapamide on macrovascular and microvascular outcomes in patients with type 2 diabetes mellitus (the ADVANCE trial): a randomised controlled trial. Lancet 370(9590): 829-840. https://doi.org/10.1016/S0140-6736(07)61303-8

12. Patel A, MacMahon S, Chalmers J et al (2008) Intensive blood glucose control and vascular outcomes in patients with type 2 diabetes. N Eng J of Med 358(24):2560-2572. https://doi.org/10. 1056/NEJMoa0802987

13. Hillis GS, Welsh P, Chalmers J et al (2014) The relative and combined ability of high-sensitivity cardiac troponin $\mathrm{T}$ and $\mathrm{N}$ terminal pro-B-type natriuretic peptide to predict cardiovascular events and death in patients with type 2 diabetes. Diabetes Care 37(1):295-303. https://doi.org/10.2337/dc13-1165

14. Würtz P, Kangas AJ, Soininen P, Lawlor DA, Davey Smith G, AlaKorpela M (2017) Quantitative serum nuclear magnetic resonance metabolomics in large-scale epidemiology: a primer on -omic technologies. A J Epidemiol 186(9):1084-1096. https://doi.org/10. 1093/aje/kwx016

15. Soininen P, Kangas AJ, Würtz P et al (2009) High-throughput serum NMR metabonomics for cost-effective holistic studies on systemic metabolism. Analyst 134(9):1781-1785. https://doi.org/ 10.1039/b910205a

16. Soininen P, Kangas AJ, Würtz P, Suna T, Ala-Korpela M (2015) Quantitative serum nuclear magnetic resonance metabolomics in cardiovascular epidemiology and genetics. Cir Cardiovasc Genet 8(1):192-206. https://doi.org/10.1161/CIRCGENETICS.114. 000216

17. Harrell FEJ (2001) Regression modeling strategies. Springer, New York. https://doi.org/10.1007/978-1-4757-3462-1

18. Prentice RL, Marek P (1979) A qualitative discrepancy between censored data rank tests. Biometrics 35(4):861-867. https://doi. org/10.2307/2530120

19. Kim Y, Kong L (2015) Estimation of C-index for Cox proportional hazards model with censored biomarker covariate subject to limits of detection. J Biopharm Stat 25(3):459-473. https://doi.org/10. 1080/10543406.2014.920859

20. Zheng Y, Parast L, Cai T, Brown M (2013) Evaluating incremental values from new predictors with net reclassification improvement in survival analysis. Lifetime Data Anal 19(3):350-370. https://doi. org/10.1007/s10985-012-9239-z

21. Sun Q, Ma J, Campos H et al (2008) Blood concentrations of individual long-chain n-3 fatty acids and risk of nonfatal myocardial infarction. A Am J Clin Nutrition 88(1):216-223. https://doi. org/10.1093/ajen/88.1.216

22. Mozaffarian D, Lemaitre RN, King IB et al (2013) Plasma phospholipid long-chain omega-3 fatty acids and total and causespecific mortality in older adults: a cohort study. Ann Intern Med 158(7):515-525. https://doi.org/10.7326/0003-4819-158-7201304020-00003

23. Lemaitre RN, King IB, Mozaffarian D, Kuller LH, Tracy RP, Siscovick DS (2003) n-3 polyunsaturated fatty acids, fatal ischemic heart disease, and nonfatal myocardial infarction in older adults: the 
Cardiovascular Health Study. Am J Clin Nutr 77(2):319-325. https://doi.org/10.1093/ajen/77.2.319

24. Matthan NR, Ooi EM, Van Horn L, Neuhouser ML, Woodman R, Lichtenstein AH (2014) Plasma phospholipid fatty acid biomarkers of dietary fat quality and endogenous metabolism predict coronary heart disease risk: a nested case-control study within the Women's Health Initiative observational study. J Am Heart Assoc 3(4). https://doi.org/10.1161/JAHA.113.000764

25. Albert CM, Campos H, Stampfer MJ et al (2002) Blood levels of long-chain n-3 fatty acids and the risk of sudden death. Eng J Med 346(15):1113-1118. https://doi.org/10.1056/NEJMoa012918

26. Simon JA, Hodgkins ML, Browner WS, Neuhaus JM, Bernert JT Jr, Hulley SB (1995) Serum fatty acids and the risk of coronary heart disease. Am J Epidemiol 142(5):469-476. https://doi.org/10. 1093/oxfordjournals.aje.a117662

27. Aung T, Halsey J, Kromhout D et al (2018) Associations of omega3 fatty acid supplement use with cardiovascular disease risks: metaanalysis of 10 trials involving 77917 individuals. JAMA Cardiol 3(3):225-234. https://doi.org/10.1001/jamacardio.2017.5205

28. Siscovick DS, Barringer TA, Fretts AM et al (2017) Omega-3 polyunsaturated fatty acid (fish oil) supplementation and the prevention of clinical cardiovascular disease: a science advisory from the American Heart Association. Circulation 135(15):e867-e884. https://doi.org/10.1161/CIR.0000000000000482

29. Elagizi A, Lavie CJ, Marshall K, DiNicolantonio JJ, O'Keefe JH, Milani RV (2018) Omega-3 polyunsaturated fatty acids and cardiovascular health: a comprehensive review. Progress in Cardiovasc Dis 61(1):76-85. https://doi.org/10.1016/j.pcad.2018.03.006

30. O'Keefe EL, Harris WS, DiNicolantonio JJ et al (2019) Sea change for marine omega-3s: randomized trials show fish oil reduces cardiovascular events. Mayo Clin Proc 94(12):2524-2533. https:// doi.org/10.1016/j.mayocp.2019.04.027

31. The ASCEND Study Collaborative Group (2018) Effects of n-3 fatty acid supplements in diabetes mellitus. New Eng J Med 379(16):1540-1550. https://doi.org/10.1056/NEJMoa1 804989

32. Oikawa S, Yokoyama M, Origasa H et al (2009) Suppressive effect of EPA on the incidence of coronary events in hypercholesterolemia with impaired glucose metabolism: sub-analysis of the Japan EPA Lipid Intervention Study (JELIS). Atherosclerosis 206(2):535-539. https://doi.org/10.1016/j.atherosclerosis.2009.03.029

33. Tavazzi L, Maggioni AP, Marchioli R et al (2008) Effect of $n-3$ polyunsaturated fatty acids in patients with chronic heart failure (the GISSI-HF trial): a randomised, double-blind, placebocontrolled trial. Lancet 372(9645):1223-1230. https://doi.org/10. 1016/S0140-6736(08)61239-8

34. Kromhout D, Geleijnse JM, de Goede J et al (2011) N-3 fatty acids, ventricular arrhythmia-related events, and fatal myocardial infarction in postmyocardial infarction patients with diabetes. Diabetes Care 34(12):2515-2520. https://doi.org/10.2337/dc11-0896

35. Tajuddin N, Shaikh A, Hassan A (2016) Prescription omega-3 fatty acid products: considerations for patients with diabetes mellitus. Diabetes Metab Syndr Obes 9:109-118. https://doi.org/10.2147/ DMSO.S97036

36. Hartweg J, Farmer AJ, Perera R, Holman RR, Neil HA (2007) Meta-analysis of the effects of $n-3$ polyunsaturated fatty acids on lipoproteins and other emerging lipid cardiovascular risk markers in patients with type 2 diabetes. Diabetologia 50(8):1593-1602. https://doi.org/10.1007/s00125-007-0695-z

37. De Luis DA, Conde R, Aller R et al (2009) Effect of omega-3 fatty acids on cardiovascular risk factors in patients with type 2 diabetes mellitus and hypertriglyceridemia: an open study. Eur Rev for Med Pharmacol Sci 13(1):51-55

38. Hendrich S (2010) (n-3) Fatty acids: clinical trials in people with type 2 diabetes. Adv Nutr 1(1):3-7. https://doi.org/10.3945/an.110. 1003

39. Mozaffarian D, Rimm EB (2006) Fish intake, contaminants, and human health: evaluating the risks and the benefits. JAMA 296(15): 1885-1899. https://doi.org/10.1001/jama.296.15.1885

40. Mozaffarian D, Wu JH (2011) Omega-3 fatty acids and cardiovascular disease: effects on risk factors, molecular pathways, and clinical events. J Am Coll Cardiol 58(20):2047-2067. https://doi.org/ 10.1016/j.jacc.2011.06.063

41. Rimm EB, Appel LJ, Chiuve SE et al (2018) Seafood long-chain n3 polyunsaturated fatty acids and cardiovascular disease: a science advisory from the American Heart Association. Circulation 138(1): e35-e47. https://doi.org/10.1161/CIR.0000000000000574

42. Lelli D, Antonelli Incalzi R, Ferrucci L, Bandinelli S, Pedone C (2020) Association between PUFA intake and serum concentration and mortality in older adults: a cohort study. Clin Nutr 39(2):510 515. https://doi.org/10.1016/j.clnu.2019.02.030

43. Wang L, Folsom AR, Eckfeldt JH (2003) Plasma fatty acid composition and incidence of coronary heart disease in middle aged adults: the Atherosclerosis Risk in Communities (ARIC) study. Nutr, Metab Cardiovasc Dis 13(5):256-266. https://doi.org/10.1016/ S0939-4753(03)80029-7

44. Marklund M, Wu JHY, Imamura F et al (2019) Biomarkers of dietary omega- 6 fatty acids and incident cardiovascular disease and mortality. Circulation 139(21):2422-2436. https://doi.org/10. 1161/circulationaha.118.038908

45. Clarke R, Shipley M, Armitage J, Collins R, Harris W (2009) Plasma phospholipid fatty acids and CHD in older men: Whitehall study of London civil servants. Br J Nutr 102(2):279284. https://doi.org/10.1017/S0007114508143562

46. Würtz P, Havulinna AS, Soininen P et al (2015) Metabolite profiling and cardiovascular event risk: a prospective study of 3 population-based cohorts. Circulation 131(9):774-785. https://doi. org/10.1161/CIRCULATIONAHA.114.013116

47. Lauretani F, Semba RD, Bandinelli S et al (2008) Plasma polyunsaturated fatty acids and the decline of renal function. Clinical Chemistry 54(3):475-481. https://doi.org/10.1373/clinchem.2007. 095521

48. Block R, Kakinami L, Liebman S, Shearer GC, Kramer H, Tsai M (2012) Cis-vaccenic acid and the Framingham risk score predict chronic kidney disease: the Multi-ethnic Study of Atherosclerosis (MESA). Prostaglandins Leukot Essent Fatty Acids 86(4-5):175182. https://doi.org/10.1016/j.plefa.2012.02.009

49. Anton G, Wilson R, Yu ZH et al (2015) Pre-analytical sample quality: metabolite ratios as an intrinsic marker for prolonged room temperature exposure of serum samples. PLoS One 10(3): e0121495. https://doi.org/10.1371/journal.pone.0121495

50. Schutte BAM, van den Akker EB, Deelen J et al (2016) The effect of standardized food intake on the association between BMI and 1H-NMR metabolites. Scientific Reports 6(1):38980. https://doi. org $/ 10.1038 /$ srep38980

Publisher's note Springer Nature remains neutral with regard to jurisdictional claims in published maps and institutional affiliations. 